\title{
Correction
}

\section{Correction to: Regularity properties of some perturbations of non-densely defined operators with applications}

\section{DELIANG CHEND}

\section{Correction to: J. Evol. Equ.}

\section{https://doi.org/10.1007/s00028-019-00510-y}

The original publication of the article contains errors which need to be amended as mentioned below:

In Sect. 6.2. "Standard assumptions and main results", line 9, the definition of $\mathcal{A}$ is obviously wrong (since for $\varphi \in D\left(\mathcal{B}_{0}\right)$ one must have $\varphi(0)=0$ which makes $\mathcal{A}$ useless). Hence, the following lines 8-11:

Set $X=E \times L^{p}((0, c), E), X_{0}=\{0\} \times L^{p}((0, c), E)$,

$$
\mathcal{A}: D(\mathcal{A})=\{0\} \times D\left(\mathcal{B}_{0}\right) \rightarrow X:(0, \varphi) \mapsto\left(-\varphi(0), \mathcal{B}_{0} \varphi\right)
$$

(here $\mathcal{A}$ is well defined due to $D\left(\mathcal{B}_{0}\right) \subset C([0, c), E)$ ) and

$$
\mathcal{L}: X_{0} \rightarrow X,(0, \varphi) \mapsto(L \varphi, 0), L \varphi=\int_{0}^{c} C(a) \varphi(a) \mathrm{d} a .
$$

should be replaced with:

Set $X=E \times L^{p}((0, c), E), X_{0}=\{0\} \times L^{p}((0, c), E)$, and

$$
\mathcal{L}: X_{0} \rightarrow X,(0, \varphi) \mapsto(L \varphi, 0), L \varphi=\int_{0}^{c} C(a) \varphi(a) \mathrm{d} a .
$$

Moreover, there is a unique closed operator $\mathcal{A}$ with $\overline{D(\mathcal{A})}=X_{0}$ such that for all $(y, f) \in X$ and $\lambda \in \rho\left(\mathcal{B}_{0}\right)$, $R(\lambda, \mathcal{A})(y, f)=(0, \varphi)$, where

$$
\varphi(a)=e^{-\lambda a} U(a, 0) y+\left(R\left(\lambda, \mathcal{B}_{0}\right) f\right)(a), a \in(0, c),
$$

see [29, Lemma 6.2], [47] contains more descriptions of $\mathcal{A}$.

The original article can be found online at https://doi.org/10.1007/s00028-019-00510-y. 
Now, $\mathcal{A}_{X_{0}}=0 \times \mathcal{B}_{0}\left(\right.$ as $\left.R\left(\lambda, \mathcal{A}_{X_{0}}\right)=\left.R(\lambda, \mathcal{A})\right|_{X_{0}}=R\left(\lambda, \mathcal{B}_{0}\right)\right)$ and Eq. (6.4) are true, and so all the following results and proofs remain unchanged.

In Lemma 2.4, last line: "Others follow from (b) and Definition 2.3" should be "Others follow from (b) and Lemma 2.3".

In Definition 6.3, line 2: " $\mathcal{B}_{0}$ is an invertible closure of" should be " $\mathcal{B}_{0}$ is a closure of".

In Example 6.13, line 5: "with $\sup _{0<a<c}\{|A(a)|,|C(a)|\}<\infty$ " should be "with $\sup _{0<a<c}\{|A(a)|\}<\infty$ and $|C(\cdot)| \in L^{p^{\prime}}(0, c)\left(1 / p^{\prime}+1 / p=1\right)$ ".

Publisher's Note Springer Nature remains neutral with regard to jurisdictional claims in published maps and institutional affiliations.

\author{
Deliang Chen \\ Department of Mathematics \\ Shanghai Jiao Tong University \\ Shanghai 200240 \\ People's Republic of China \\ E-mail: chernde@sjtu.edu.cn
}

